\title{
Therapeutic plasma exchange as an effective salvage measure for post-hepatectomy hepatic failure: A case report
}

\author{
Lee $\mathrm{Na}$ Ryu, Shin Hwang, and Suhyeon $\mathrm{Ha}$
}

Department of Surgery, Asan Medical Center, University of Ulsan College of Medicine, Seoul, Korea

\begin{abstract}
Major hepatectomy can result in post-hepatectomy hepatic failure (PHHF) and therapeutic plasma exchange (TPE) can be used as a salvage procedure for liver support. We herein present a case of 69-year-old male patient with perihilar cholangiocarcinoma who was successfully managed with salvage TPE. Preoperative portal embolization was performed to reduce the parenchymal resection rate. The extent of surgery was right hepatectomy with partial excision of the ventral portion of the segment IV, caudate lobectomy, bile duct resection and extensive lymph node dissection. No noticeable surgical complications occurred after the operation, but serum total bilirubin level increased gradually and reached $10 \mathrm{mg} / \mathrm{dl}$ at 1 month after the operation. At postoperative day 38, total bilirubin level raised to $19.8 \mathrm{mg} / \mathrm{dl}$ and prothrombin time deteriorated significantly, thus salvage TPE was started. TPE was performed three times per week for 2 weeks; consequently, the total bilirubin level was maintained below $10 \mathrm{mg} / \mathrm{dl}$. A few days later, a rebound of total bilirubin occurred; accordingly, 2 sessions of TPE were performed additionally. Overall, a total of 8 sessions of TPE were performed. The patient was discharged at 84 days after operation. The total bilirubin level returned to normal at 5 months after operation. This patient is doing well for past 9 months. In Korea, TPE for liver support has been approved by the social health insurance since August 2020. In conclusion, salvage TPE is an effective liver support measure for PHHF, thus we suggest starting TPE if serum total bilirubin level reaches $10 \mathrm{mg} / \mathrm{dl}$ after hepatectomy. (Ann Hepatobiliary Pancreat Surg 2021;25:270-275)
\end{abstract}

Key Words: Major hepatectomy; Hepatic failure; Hyperbilirubinemia; Liver support; Hepatic decompensation

\section{INTRODUCTION}

Major hepatectomy of the diseased liver can result in hepatic decompensation and even fatal hepatic failure. To minimize such operative risks, the hepatic reserve is assessed preoperatively, including both qualitative and quantitative aspects of the liver function. Although when these preoperative parameters appear to permit major hepatectomy, there exists a possibility of post-hepatectomy hepatic failure (PHHF). ${ }^{1-3}$ Once PHHF occurs after liver resection, there is nearly no effective treatment modality for the prevention of additional aggravation except for general liver support. We have previously reported a case of a patient with PHHF after right hepatectomy who was successfully managed by therapeutic plasma exchange (TPE). ${ }^{4}$ We have occasionally performed TPE for patients waiting for liver transplantation (LT) as a bridge therapy to LT and for those showing early allograft dysfunction as a liver function support. ${ }^{5-7}$ Nevertheless, TPE has been rarely performed for patients with PHHF because of its rare incidence. We herein present the case of a patient with PHHF after right hepatectomy who was successfully managed by salvage TPE.

\section{CASE}

The patient was a 69-year-old male with perihilar cholangiocarcinoma. He was admitted to our institution due to recent body weight loss and jaundice. Imaging studies led to the diagnosis of perihilar cholangiocarcinoma of Bismuth type IV, with abutting the right hepatic artery and focal attachment to the left portal vein and middle hepatic artery (Fig. 1). The patient was not associated with viral hepatitis, but had a history of heavy alco-

Received: October 29, 2020; Revised: November 5, 2020; Accepted: November 6, 2020

Corresponding author: Shin Hwang

Department of Surgery, Asan Medical Center, University of Ulsan College of Medicine, 88 Olympic-ro 43-gil, Songpa-gu, Seoul 05505, Korea Tel: +82-2-3010-3930, Fax: +82-2-3010-6701, E-mail: shwang@amc.seoul.kr

Copyright (C) 2021 by The Korean Association of Hepato-Biliary-Pancreatic Surgery

This is an Open Access article distributed under the terms of the Creative Commons Attribution Non-Commercial License (http://creativecommons.org/ licenses/by-nc/4.0) which permits unrestricted non-commercial use, distribution, and reproduction in any medium, provided the original work is properly cited. Annals of Hepato-Biliary-Pancreatic Surgery • pISSN: 2508-5778 - eISSN: 2508-5859 

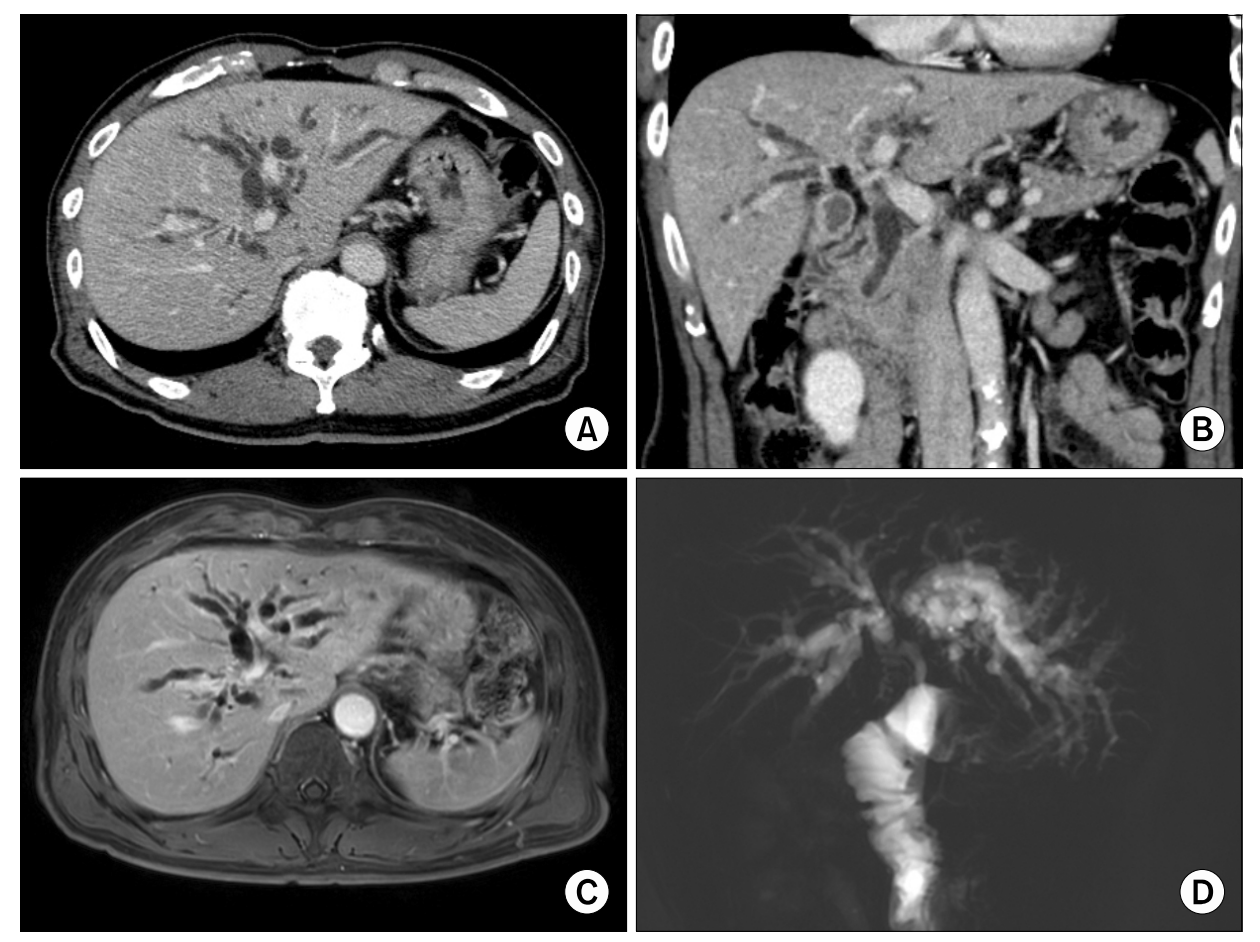

Fig. 1. Initial preoperative radiologic findings. Bismuth type IV perihilar cholangiocarcinoma is visible at the dynamic abdomen computed tomography images (A, B) and magnetic resonance cholangiopancreatography $(\mathrm{C}, \mathrm{D})$.
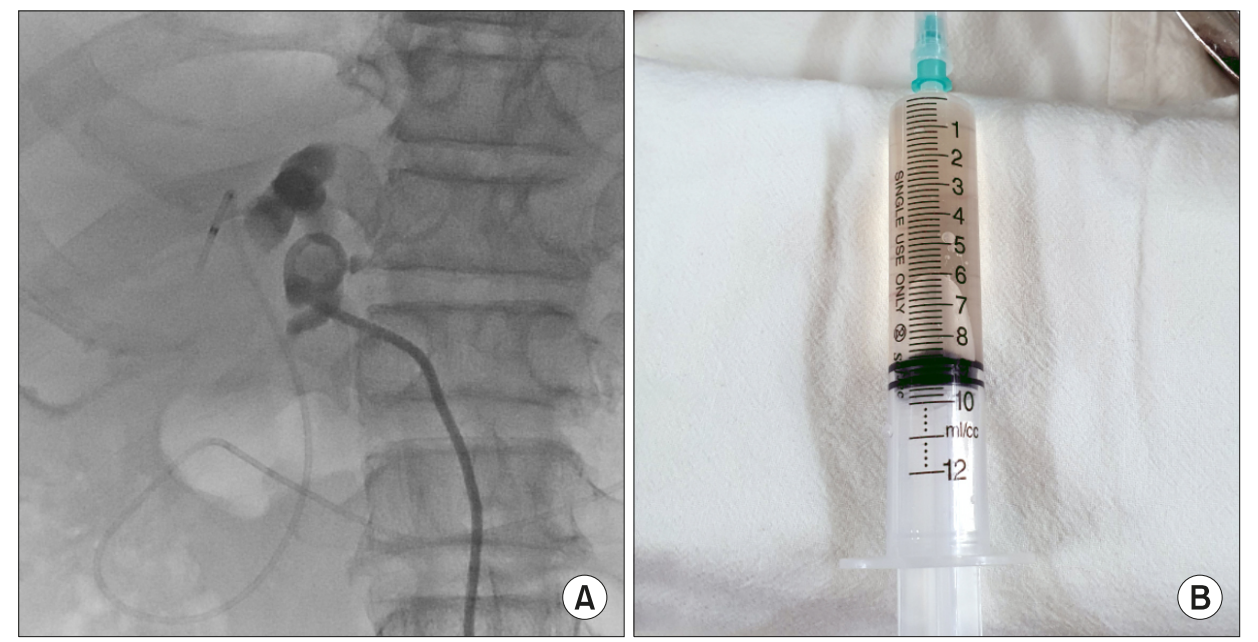

Fig. 2. Preoperative percutaneous biliary drainage (PTBD). (A) A PTBD catheter was inserted into the left hepatic duct. (B) The color of bile from the left hepatic duct appears watery clear, indicating loss of excretory hepatocyte function.

hol intake for a long period. Endoscopic retrograde cholangiography was performed for biliary decompression through the endoscopic nasobiliary drainage (ENBD). Endoscopic biopsy of the hilar bile duct revealed moderately differentiated adenocarcinoma. The initial serum total bilirubin level was $10.8 \mathrm{mg} / \mathrm{dl}$, but no decrease was noted for 4 days following ENBD into the right hepatic duct. For further biliary decompression, percutaneous biliary drainage (PTBD) was performed through the left hepatic duct. The color of bile drained from the left hepatic duct appeared watery clear, indicating loss of excretory hepatocyte function (Fig. 2). The serum bilirubin level de- creased slowly to $3.8 \mathrm{mg} / \mathrm{dl}$ at 14 days after biliary decompression with ENBD and PTBD.

Although the volume proportion of the future remnant left liver was $39 \%$ of the whole liver, we considered that this patient was at high risk of PHHLF because of the very slow resolution of obstructive jaundice. As we planned to perform right hepatectomy, percutaneous transhepatic portal embolization (PTPE) was performed to induce compensatory hypertrophy of the future remnant left liver and atrophy of the right liver. After waiting for 20 days, the future remnant left liver appeared as $50 \%$ of the whole liver volume (Fig. 3), thus we decided to perform 

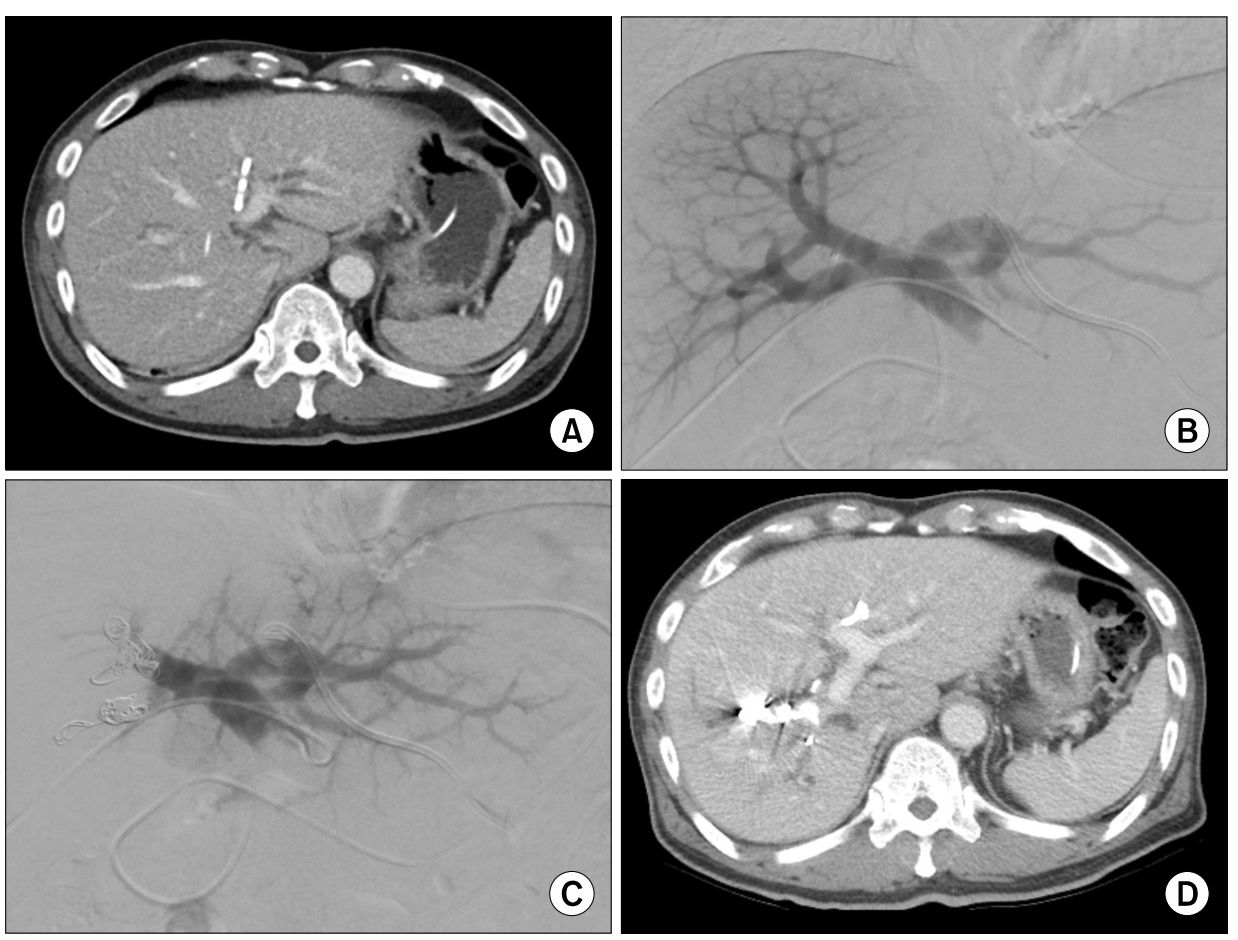

Fig. 3. Imaging study findings before and after percutaneous transhepatic portal embolization (PTPE). (A) The pre-PTPE volume of the future remnant left liver is $39 \%$ of the whole liver volume. $(\mathrm{B}, \mathrm{C})$ Right PTPE is performed through an ipsilateral approach. (D) The future remnant left liver is increased to $50 \%$ of the whole liver volume at 20 days after PTPE.

right hepatectomy, caudate lobectomy and bile duct resection.

The operation was performed at 22 days after PTPE and the patient's total bilirubin concentration was 2.0 $\mathrm{mg} / \mathrm{dl}$. The right liver showed noticeable wrinkling with chronic hepatitis background, which made the interlobar demarcation noticeable. The extent of surgery was right hepatectomy with partial excision of the ventral portion of the segment IV, caudate lobectomy, bile duct resection and extensive lymph node dissection. After mobilization of the right liver, the hepatoduodenal ligament was dissected. After confirming that the resection margin of the distal bile duct was tumor-negative on the frozen-section biopsy, the right hepatic artery was clamped with a bulldog clamp, that induced hemihepatic inflow block because the right portal vein was embolized already. The liver parenchyma was transected with a Cavitron ultrasonic surgical aspirator and electrocautery. The clamping of the hepatic inflow to the left liver was performed for only 15 minutes. As intraoperative blood loss was estimated to be $500 \mathrm{ml}$, blood transfusion was not required. Roux-en-Y hepaticojejunostomy was performed after unification of two left hepatic duct openings. The left PTBD catheter was not removed for later monitoring of the liver function because the patient showed slow biliary decom-

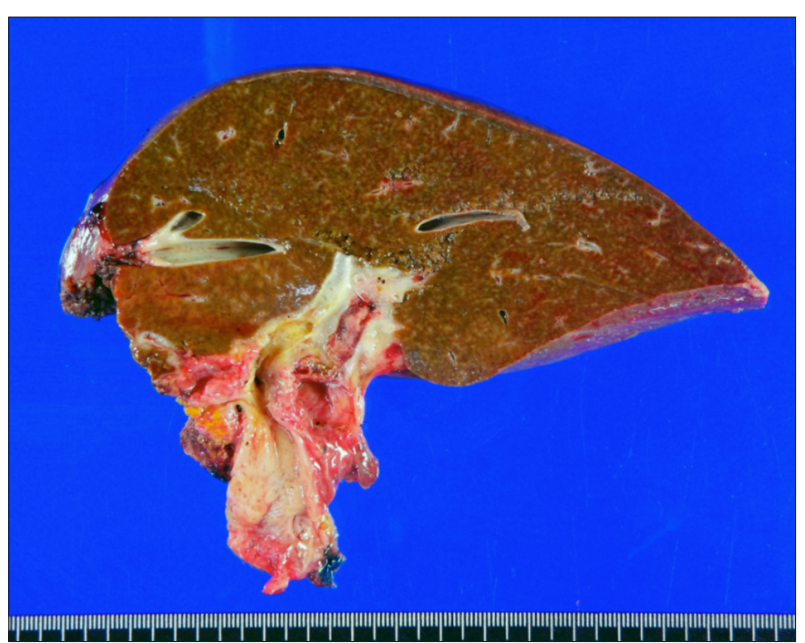

Fig. 4. Gross photograph of the resected specimen showing a perihilar cholangiocarcinoma of flat infiltrative type, 4.2 $\mathrm{cm}$-sized moderately differentiated adenocarcinoma, with extension beyond the bile duct and involvement of the liver.

pression indicating impaired functional reserve of the liver.

The pathology report of the resected specimen showed that the tumor was perihilar cholangiocarcinoma of flat infiltrative type, $4.2 \mathrm{~cm}$-sized moderately differentiated adenocarcinoma, with extension beyond the bile duct and involvement of the liver (Fig. 4). The depth of tumor invasion was $5 \mathrm{~mm}$ from surface epithelia. Lymphovascular 

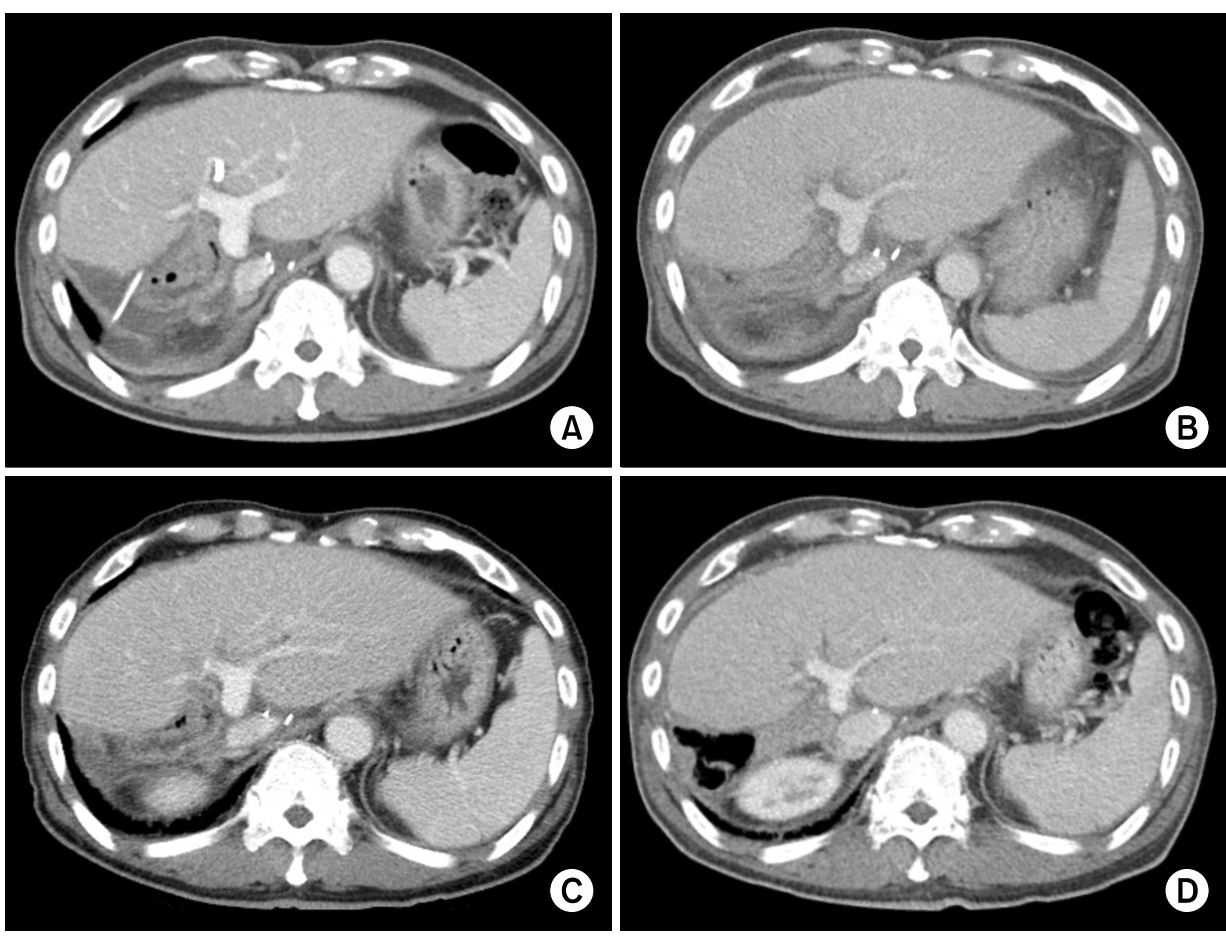

Fig. 5. Postoperative computed tomography (CT) follow-up images. No abnormal findings can be seen in the 1-week CT scan (A). One-month CT scan shows ascites and splenomegaly (B). No abnormal findings except splenomegaly are visible in the 3-month (C) and 8-month (D) CT scans.

invasion and perineural invasion were present. There was no tumor metastasis in 9 resected lymph nodes. The liver parenchyma showed bridging fibrosis on the Masson trichrome staining. According to the 8th edition of the American Joint Committee on Cancer (AJCC), this tumor was identified as T2aN0M0, thus being stage II.

No noticeable surgical complications occurred after the operation (Fig. 5A). However, the serum total bilirubin level increased gradually and reached a stationary state level of $10 \mathrm{mg} / \mathrm{dl}$ at 1 month after operation (Fig. 6). At that time, the levels of blood tests were as follows: serum aspartate transaminase (AST) $83 \mathrm{IU} / \mathrm{L}$, alanine transaminase (ALT) $61 \mathrm{IU} / \mathrm{L}$, total bilirubin $10.3 \mathrm{mg} / \mathrm{dl}$, albu$\min 2.8 \mathrm{~g} / \mathrm{dl}$, total cholesterol $98 \mathrm{mg} / \mathrm{dl}$, prothrombin time $58.6 \%$ (1.32 INR), and platelet count $125 \times 10^{3} / \mu 1$. CT scan showed a moderate amount of ascites (Fig. 5B), thus repeated albumin infusion with diuretics therapy was continued. As the general condition of the patient deteriorated slightly, we continued intravenous hyperalimentation and oral intake was encouraged. There was no evidence of surgical complications, and biliary tubogram through the remained PTBD catheter showed the good passage through the hepaticojejunostomy.

We continued supportive care for post-hepatectomy hyperbilirubinemia for more than 1 month, but the total bi-

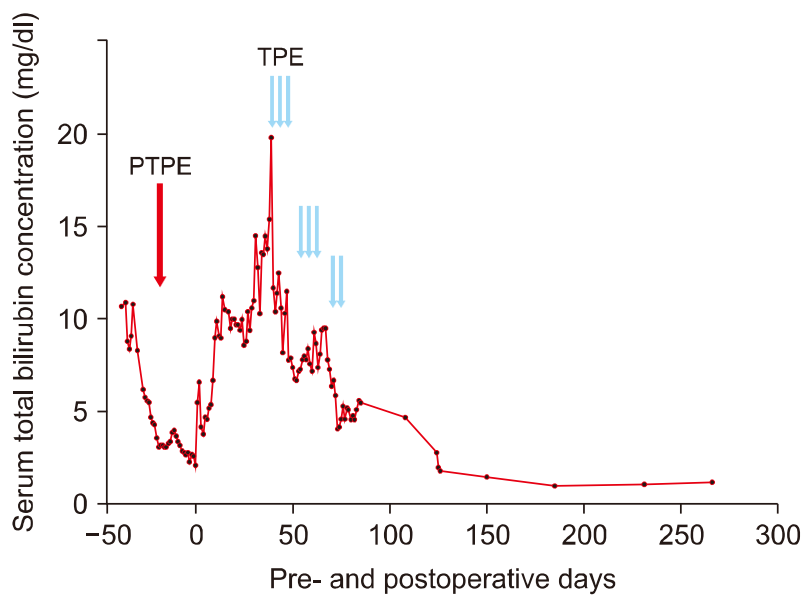

Fig. 6. Changes in the serum total bilirubin level before and after surgery. A total of 8 sessions of therapeutic plasma exchange (TPE) were performed. PTPE denotes percutaneous transhepatic portal embolization.

lirubin level raised to $19.8 \mathrm{mg} / \mathrm{dl}$ and prothrombin time INR deteriorated further at postoperative day 38. On the same day, TPE was started. TPE was performed three times per week for 2 weeks; subsequently total bilirubin level was maintained below $10 \mathrm{mg} / \mathrm{dl}$. A few days later, as a rebound of total bilirubin occurred, 2 additional sessions of TPE were performed. Thereafter, the total bilirubin level was lowered below $5 \mathrm{mg} / \mathrm{dl}$ and decreased gradually over 3 months (Fig. 6). The patient was dis- 
charged at 84 days after the operation. The total bilirubin level returned to normal after 5 months of operation and no abnormal findings were identified at the follow-up CT scans (Fig. 5C, D). Currently, the patient is doing well for the past 9 months, without tumor recurrence or deterioration of liver function.

\section{DISCUSSION}

Progressive deterioration of liver function after major hepatectomy usually lead to fatal sequences. PHHF usually shows hyperbilirubinemia, coagulopathy and hepatic encephalopathy. Its pathophysiology is unknown, but it is reported that PHHF is mediated by postoperative portal hyperperfusion into the small liver remnant which leads to transient portal hypertension, sinusoidal constriction and endothelial injury. ${ }^{8}$ Risk factors of the development of PHHF include an excessively high rate of liver resection that induces small remnant liver volume, underlying liver cirrhosis, steatosis, advanced age, malnutrition, diabetes, and the existence of preoperative portal hypertension. ${ }^{9,10}$ Infection has been reported to complicate the course of treatment as a precipitant or during later stages due to the reduced phagocytosis capacity of the hepatic reticuloendothelial system. ${ }^{9}$ Bacterial translocation from prolonged fasting and diminished hepatic clearance of bacteria might enhance the susceptibility for the development of infections. ${ }^{11}$

To improve the sequences of PHHF, liver function support during the acute phase of hepatic function deterioration appears to be effective. Various blood purification therapies have been developed for the treatment of acute liver failure. ${ }^{10}$ We previously reported our experience on the use of TPE and Molecular Adsorbent Recirculating System (MARS) in patients with posttransplant graft dysfunction. $^{7}$ After a single MARS session, patients showed a significant reduction in the levels of creatinine, urea nitrogen, bilirubin, and ammonia. After a single TPE session, patients showed a significant improvement in the levels of prothrombin time, total bilirubin, ALT, alkaline phosphatase, and albumin. After the completion of treatment, both MARS and TPE led to significant improvement in the bilirubin levels. Regarding PHHF, TPE is considered as more suitable as a supportive measure.

TPE as a method of plasma exchange to replace fresh plasma after separation from blood has demonstrated encouraging results in selected patients. ${ }^{12-14}$ In our present patient, soon after salvage TPE, serum total bilirubin began to decrease probably due to initial dilution effect and restoration of hepatic metabolic capability. The sessions of TPE were repeated until a marked decrease in serum total bilirubin level was attained. Besides the total bilirubin level, we focused on the changes in the total cholesterol levels and prothrombin time. We previously reported that a rise in total cholesterol level can indicate recovery of hepatic function. ${ }^{4}$ Hypocholesterolemia has been found to be an independent predictor of mortality in critical surgical illnesses. ${ }^{15,16}$ Notably cholesterol is produced only by the liver. ${ }^{17}$ Temporary liver support using TPE may induce the functional recovery of a failing liver. Prothrombin time also showed a similar pattern of recovery as the serum cholesterol level. Initial improvement in prothrombin time could have been due to coagulation factor replacement through plasmapheresis per se, but its recovery pattern was indicative of the rapid recovery of hepatic synthetic function. It is known that parenteral vitamin $\mathrm{K}$ replacement corrects coagulopathy related to biliary obstruction, bacterial overgrowth or malnutrition, ${ }^{18}$ thus we frequently administered vitamin K1 for patients with obstruction jaundice and impaired coagulation profiles.

On the contrary to serum cholesterol and prothrombin time, the decrease in serum total bilirubin concentration was often delayed in our previous case and the present case. $^{4}$ TPE was continued until the disappearance of the rebound rise in serum bilirubin was achieved, because the initial and temporary decrease in bilirubin concentration was probably due to the blood dilution effect and its small therapeutic effect.

Although we knew the beneficial effect of TPE, the initiation of TPE was significantly delayed because TPE for liver support was not financially approved until July 2020 by the Health Insurance Review and Assessment Service in Korea. TPE has been approved as a useful procedure for PHHF and graft dysfunction since August 2020, in which the first 3 sessions of TPE are fully covered and the next sessions are half covered by the social health insurance in Korea. This recent approval of TPE is beneficial to perform TPE at an early stage and more frequently than before.

There exists a randomized controlled trial on TPE in patients with acute liver failure, ${ }^{19}$ in which treatment with 
TPE has led to the improvement in the outcome in patients with acute liver failure by increasing liver transplant-free survival. This is attributable to the attenuation of innate immune activation and amelioration of multi-organ dysfunction. A Japanese single-center study suggested that TPE improved liver function in living donor liver transplantation recipients with progressive liver failure and appears to be indicated in patients with total bilirubin levels ranging between 13 and $24 \mathrm{mg} / \mathrm{dl}^{20}$

There exist no practical guidelines to start TPE for liver support in the literature. The task force team for TPE in the Korean Association of Hepato-Biliary-Pancreatic Surgery and the Korean Society for Transplantation recommends that TPE is eligibly indicated if the total serum bilirubin level exceeds $10 \mathrm{mg} / \mathrm{dl}$ after hepatectomy or liver transplantation and TPE sessions can be repeated every 2 or 3 other days until loss of rebound rise in serum total bilirubin level is achieved.

In conclusion, salvage TPE is a useful liver support for PHHF, thus we suggest starting TPE if serum total bilirubin level reaches $10 \mathrm{mg} / \mathrm{dl}$ after hepatectomy.

\section{CONFLICT OF INTEREST}

None of the authors has any conflict of interest.

\section{ORCID}

Lee Na Ryu: https://orcid.org/0000-0002-2868-3355

Shin Hwang: https://orcid.org/0000-0002-9045-2531

Suhyeon Ha: https://orcid.org/0000-0001-8234-4170

\section{AUTHOR CONTRIBUTIONS}

Conceptualization: SH. Data curation: LNR, SH, SHa. Formal analysis: SH. Methodology: LNR, SHa. Project administration: SH. Visualization: SH. Writing - original draft: LNR, SH. Writing - review \& editing: All.

\section{REFERENCES}

1. Lee SG, Hwang S. How I do it: assessment of hepatic functional reserve for indication of hepatic resection. J Hepatobiliary Pancreat Surg 2005;12:38-43.

2. Shirabe K, Shimada M, Gion T, Hasegawa H, Takenaka K,
Utsunomiya $\mathrm{T}$, et al. Postoperative liver failure after major hepatic resection for hepatocellular carcinoma in the modern era with special reference to remnant liver volume. J Am Coll Surg 1999; 188:304-309.

3. Hwang S, Ha TY, Song GW, Jung DH, Ahn CS, Moon DB, et al. Quantified risk assessment for major hepatectomy via the indocyanine green clearance rate and liver volumetry combined with standard liver volume. J Gastrointest Surg 2015;19:1305-1314.

4. Hwang S, Ha TY, Ahn CS, Kim KH, Lee SG. Reappraisal of plasmapheresis as a supportive measure in a patient with hepatic failure after major hepatectomy. Case Rep Gastroenterol 2007;1: 162-167.

5. Choe W, Kwon SW, Kim SS, Hwang S, Song GW, Lee SG. Effects of therapeutic plasma exchange on early allograft dysfunction after liver transplantation. J Clin Apher 2017;32:147-153.

6. Park CS, Hwang S, Park HW, Park YH, Lee HJ, Namgoong JM, et al. Role of plasmapheresis as liver support for early graft dysfunction following adult living donor liver transplantation. Transplant Proc 2012;44:749-751.

7. Lee JY, Kim SB, Chang JW, Park SK, Kwon SW, Song KW, et al. Comparison of the molecular adsorbent recirculating system and plasmapheresis for patients with graft dysfunction after liver transplantation. Transplant Proc 2010;42:2625-2630.

8. Lafaro K, Buettner S, Maqsood H, Wagner D, Bagante F, Spolverato $\mathrm{G}$, et al. Defining post hepatectomy liver insufficiency: where do we stand? J Gastrointest Surg 2015;19:2079-2092.

9. Kauffmann R, Fong Y. Post-hepatectomy liver failure. Hepatobiliary Surg Nutr 2014;3:238-246.

10. Naruse K, Nagashima H, Sakai Y, Kokudo N, Makuuchi M. Development and perspectives of perfusion treatment for liver failure. Surg Today 2005;35:507-517.

11. Wells CL, Maddaus MA, Simmons RL. Proposed mechanisms for the translocation of intestinal bacteria. Rev Infect Dis 1988; 10:958-979.

12. Mandal AK, King KE, Humphreys SL, Maley WR, Burdick JF, Klein AS. Plasmapheresis: an effective therapy for primary allograft nonfunction after liver transplantation. Transplantation 2000;70:216-220.

13. Ozdemir FN, Tutal E, Sezer S, Gür G, Bilgic A, Haberal M. Effect of supportive extracorporeal treatment in liver transplantation recipients and advanced liver failure patients. Hemodial Int 2006;10 Suppl 2:S28-S32.

14. Inayat F, Hussain Q, Tasleem SH, Farooq S, Hurairah A. Salvage plasmapheresis for post-hepatectomy liver failure. Cureus 2016;8:e884.

15. Gui D, Spada PL, De Gaetano A, Pacelli F. Hypocholesterolemia and risk of death in the critically ill surgical patient. Intensive Care Med 1996;22:790-794.

16. Giovannini I, Boldrini G, Chiarla C, Giuliante F, Vellone M, Nuzzo G. Pathophysiologic correlates of hypocholesterolemia in critically ill surgical patients. Intensive Care Med 1999;25:748-751.

17. Chen J, Song W, Redinger RN. Effects of dietary cholesterol on hepatic production of lipids and lipoproteins in isolated hamster liver. Hepatology 1996;24:424-434.

18. Kaul V, Munoz SJ. Coagulopathy of liver disease. Curr Treat Options Gastroenterol 2000;3:433-438.

19. Larsen FS, Schmidt LE, Bernsmeier C, Rasmussen A, Isoniemi $\mathrm{H}$, Patel VC, et al. High-volume plasma exchange in patients with acute liver failure: an open randomised controlled trial. J Hepatol 2016;64:69-78.

20. Yamamoto R, Nagasawa Y, Marubashi S, Furumatsu Y, Iwatani $\mathrm{H}$, Iio $\mathrm{K}$, et al. Early plasma exchange for progressive liver failure in recipients of adult-to-adult living-related liver transplants. Blood Purif 2009;28:40-46. 